\title{
Outcomes of endoscopic submucosal dissection for gastric epithelial neoplasm in chronic kidney disease patients: propensity score- matched case-control analysis, methodological and statistical issues
}

\author{
Yahya Salimi $^{1,2}$ D $\cdot$ Ibrahim Abdollahpour ${ }^{3}$ (D) \\ Received: 25 September 2018 / Accepted: 12 December 2018 / Published online: 18 December 2018 \\ (c) The International Gastric Cancer Association and The Japanese Gastric Cancer Association 2018
}

Keywords Case-control $\cdot$ Propensity score $\cdot$ Standardized difference

Dear editor,

We read with interest the paper by Young Kwon Choi et al. [1] entitled "Outcomes of endoscopic submucosal dissection for gastric epithelial neoplasm in chronic kidney disease patients: propensity score-matched case-control analysis". They concluded that "ESD for gastric epithelial neoplasms can be performed in stage $3 \mathrm{CKD}$ patients with comparable efficacy and safety to that performed in non-CKD patients". Although the results seem quite interesting, there are a number of concerns which we recommend to be addressed to more clarifying the validity of the study findings.

First; for making a valid conclusion, it is critical to decrease the possibility of major selection bias when conducting a case-control study. Then, it would be better to clarify the representativeness of the study controls. Moreover, one of the critical steps in a case-control study is clarifying the study-base [2]. It would be better to clarify the study-base of cases. Did the controls come from the same study-base?

Second; some authors believe that, due to the study limitation, it would not be possible to estimate true PS from a

Ibrahim Abdollahpour

ebrahemen@gmail.com

Yahya Salimi

yahya.salimi@kums.ac.ir

1 Social Development and Health Promotion Research Center, Kermanshah University of Medical Sciences, Kermanshah, Iran

2 Department of Epidemiology, School of Public Health, Kermanshah University of Medical Sciences, Kermanshah, Iran

3 Department of Epidemiology, School of Public Health, Arak University of Medical Sciences, Arak, Iran case-control study [3]. However, Mansson et al. using simulation studies, had shown that the following methods might be practical for making a true PS estimation in a case-control study; (1) Weighted case-control, (2) Sub-cohort, (3) Control, (4) Un-weighted case-control and (5) Modeled control [4]. It is highly recommended to clarify the employed methods for PS estimation in Young Kwon Choi et al. paper.

Third; Rothman et al. has recommended some criteria for valid selection of covariates when constructing a propensity score (PS) model [5]. They has shown that "variables that are unrelated to the exposure but related to the outcome should always be included in a PS model". In contrast, due to statistical efficiency reasons, they did not support including variables that are related to the exposure, but not to the outcome [5]. The criteria recruited for covariate selection when constructing the PS model in this study have not been stated. Moreover, it would be interesting for readers to have some information regarding the utilized methods for assessing the adequacy of the final PS model.

Finally; The comparability between the study groups is a necessary criteria for making a valid conclusion regarding the validity of the study results when performing PS analysis [6]. Because of the limitation of the usual statistical test, i.e., Student's $t$ test or $\chi^{2}$ test, the standardized difference has been suggested for assessing the covariate balance between the study groups when performing the PS analysis. However, it seems that the authors have not employed an appropriate criterion for assessing the comparability between the study groups.

Without the above information, it would be optimistic to draw a valid conclusion regarding the validity of the study findings. Therefore, we recommend clarifying the enumerated issues when interpreting the study findings. 


\section{Compliance with ethical standards}

Conflict of interest The authors have no potential conflict of interest relevant to the present study.

\section{References}

1. Choi YK et al. Outcomes of endoscopic submucosal dissection for gastric epithelial neoplasm in chronic kidney disease patients: propensity score-matched case-control analysis. Gastric Cancer. 2018. https://doi.org/10.1007/s10120-018-0848-4.

2. Wacholder S, et al. Selection of controls in case-control studies: I. Principles. Am J Epidemiol. 1992;135(9):1019-28.

3. Allen AS, Satten GA. Control for confounding in case-control studies using the stratification score, a retrospective balancing score. Am J Epidemiol. 2011;173(7):752-60.
4. Månson R, et al. On the estimation and use of propensity scores in case-control and case-cohort studies. Am J Epidemiol. 2007;166(3):332-9.

5. Brookhart MA, et al. Variable selection for propensity score models. Am J Epidemiol. 2006;163(12):1149-56.

6. Austin PC. An introduction to propensity score methods for reducing the effects of confounding in observational studies. Multivar Behav Res. 2011;46(3):399-424.

Publisher's Note Springer Nature remains neutral with regard to jurisdictional claims in published maps and institutional affiliations. 